\title{
INDICADORES ANTROPOMÉTRICOS, METABÓLICOS E HEMODINÂMICOS COMO PREDITORES DA SÍNDROME METABÓLICA EM ADOLESCENTES
}

\section{Anthropometric, metabolic and hemodynamic indicators as predictors of metabolic syndrome in adolescents}

\section{Indicadores antropométricos, metabólicos y hemodinámicos como predictores del Síndrome metabólico en adolescentes}

Thais Norberta Bezerra de Moura (OrcID)

Força Estadual de Saúde do Maranhão - FESMA - São Francisco do Maranhão (MA) - Brasil

Jéssica Denise Vieira Leal (OrcID)
Universidade Federal do Piauí - UFPI - Picos (PI) - Brasil

Gisely Silva Sousa (OrcID)

Universidade Federal do Piauí - UFPI - Picos (PI) - Brasil

Regianne Kellyne Carneiro de Sousa (OrcID)

Universidade Federal do Piauí - UFPI - Picos (PI) - Brasil

\section{Edina Araújo Rodrigues Oliveira (OrcID)}

Universidade Federal do Piauí - UFPI - Picos (PI) - Brasil

Luisa Helena de Oliveira Lima (OrcID)

Universidade Federal do Piauí - UFPI - Picos (PI) - Brasil

\section{RESUMO}

Objetivo: Predizer a síndrome metabólica em adolescentes a partir de indicadores antropométricos, metabólicos e hemodinâmicos por análise de sensibilidade e especificidade. Métodos: Estudo transversal, realizado entre julho de 2015 e março de 2016 , com 186 adolescentes de oito escolas particulares do município de Picos, Piauí, Brasil. Coletaram-se os dados por meio de um formulário adaptado com informações sobre medidas antropométricas, metabólicas e pressão arterial nas dependências das escolas de forma individual em sala reservada para tal. Após um jejum noturno de 12 horas, realizou-se uma coleta de sangue venoso para posterior análise bioquímica. Utilizou-se o teste T para amostras independentes para comparação de médias dos indicadores com nível de significância de $p<0,05$. Para seleção dos pontos de corte, adotaram-se as curvas receiver operating characteristic $(R O C)$, utilizando-se os valores com sensibilidade e especificidade mais próximos entre si e não inferiores a $60 \%$. Resultados: Houve prevalência do sexo feminino $(61,8 \%$; $n=114)$, idade entre 15 a 19 anos $(57,5 \%$; $n=106)$ e da síndrome em 2,7\% ( $n=5)$ da amostra. Quando analisada a área sob a curva (AUC) ROC, encontraram-se como preditores significativos da síndrome na amostra total o índice de conicidade ( $A \cup C=0,83$ ), a lipoproteína de alta densidade $(A U C=0,88)$, a pressão arterial sistólica $(A \cup C=0,86)$ e a pressão arterial média $(A \cup C=0,84)$. Conclusão: Os indicadores investigados demonstraramse preditores da síndrome metabólica, merecendo destaque o índice de conicidade, a lipoproteína de alta densidade, a pressão arterial sistólica e a pressão arterial média.

Descritores: Síndrome Metabólica; Antropometria; Adolescente; Dislipidemias; Pressão Arterial; Obesidade Abdominal.

\section{ABSTRACT}

Objective: To predict metabolic syndrome in adolescents using anthropometric, metabolic and hemodynamic indicators based on analysis of sensitivity and specificity. Methods: Cross-sectional study carried out from July 2015 to March 2016 with 186 adolescents from eight private schools in the municipality of Picos, Piauí, Brazil. The data were individually collected using a form adapted with information on anthropometric, metabolic and blood pressure measures in a room reserved for such within the premises of the schools. After a 12-hour overnight fast, venous blood was collected for further biochemical analysis. The T-test was used for comparison of means in independent samples with significance level of $p<0.05$. Cut-off scores were selected based on the receiver operating characteristic (ROC) curves using the values with sensitivity and specificity closest to each other and 
not below 60\%. Results: There was a predominance of women (61.8\%; $n=114)$, age between 15 and 19 years $(57.5 \%$; $n=106)$ and syndrome in $2.7 \%(n=5)$ of the sample. When the area under the curve (AUC) was analyzed, conicity index (AUC=0.83), high-density lipoprotein $(A \cup C=0.88)$, systolic blood pressure $(A \cup C=0.86)$ and mean arterial pressure $(A \cup C=0.84)$ were found to be significant predictors of the syndrome in the total sample. Conclusion: The indicators analyzed proved to be predictors of metabolic syndrome, particularly conicity index, high-density lipoprotein, systolic blood pressure and mean arterial pressure.

Descriptors: Metabolic Syndrome; Anthropometry; Adolescent; Dyslipidemia; Blood Pressure; Abdominal Obesity.

\section{RESUMEN}

Objetivo: Predecir el síndrome metabólico en adolescentes a partir de los indicadores antropométricos, metabólicos y hemodinámicos por el análisis de sensibilidad y especificidad. Métodos: Estudio transversal realizado entre julio de 2015 y marzo de 2016 con 186 adolescentes de ocho escuelas privadas del municipio de Picos, Piauí, Brasil. Se recogieron los datos a través de un formulario adaptado con informaciones sobre las medidas antropométricas, metabólicas y presión arterial en las escuelas de manera individual en clase reservada para ello. Después de un ayuno nocturno de 12 horas se realizó una colecta de sangre venosa para posterior análisis bioquímico. Se utilizó el test $T$ para muestras independientes para la comparación de medias de los indicadores con el nivel de significación de $p<0,05$. Para la elección de los puntos de corte, se adoptaron las curvas receiver operating characteristic (ROC), utilizándose los valores con sensibilidad y especificidad más cercanos entre si y no inferiores al 60\%. Resultados: Hubo prevalencia para el sexo femenino $(61,8 \%$; $n=114)$, edad entre 15 y 19 años $(57,5 \%$; $n=106)$ y del síndrome en el 2,7\% $(n=5)$ de la muestra. Se han encontrado como predictores significativos para el síndrome en la muestra total el índice de conicidad $(A \cup C=0,83)$, la lipoproteina de baja densidad $(A \cup C=0,88)$, la presión arterial sistólica $(A \cup C=0,86)$ y la presión arterial media $(A \cup C=0,84)$ a partir del análisis del área bajo la curva (AUC) ROC. Conclusión: Los indicadores investigados se han demostrado predictores del síndrome metabólico con especial importancia para el índice de conicidad, la lipoproteina de alta densidad, la presión arterial sistólica y la presión arterial media.

Descriptores: Síndrome Metabólico; Antropometría; Adolescente; Dislipidemias; Presión Arterial; Obesidad Abdominal.

\section{INTRODUÇÃO}

A adolescência caracteriza-se como a fase do ciclo vital entre a infância e a fase adulta. Nesse período, o indivíduo vivencia seu estirão pôndero-estatural; o surgimento dos caracteres sexuais secundários; no caso do sexo feminino, o advento da menarca selando o início do ciclo reprodutivo; e complexas transformações no processo de desenvolvimento cognitivo, corroborando para a descoberta de novas relações intrapessoais, interpessoais e com o meio ${ }^{(1)}$.

Trata-se de um período crítico para o desenvolvimento de competências pessoais e a aquisição de habilidades para tomada de decisões, tornando-se relevante a elaboração de estratégias direcionadas à saúde dos adolescentes com foco na redução dos agravos evitáveis, fortalecendo vínculos de forma a desenvolver a autonomia dos sujeitos para o alcance da saúde com qualidade de vida(1).

A síndrome metabólica (SM) é uma agregação de problemas cardiometabólicos, sendo tida como um transtorno complexo representado por um conjunto de fatores de risco cardiovasculares usualmente relacionados à deposição de gordura e à resistência à insulina ${ }^{(2)}$.

A síndrome implica uma associação de fatores de risco capazes de predispor o indivíduo ao desenvolvimento de doenças cardiovasculares e a diabetes mellitus tipo 2, representando, desse modo, um sério problema de saúde pública da atualidade, com relevantes impactos na morbidade e na mortalidade da população ${ }^{(3)}$, sendo responsável por $7 \%$ dos óbitos globais ${ }^{(4)}$.

Os fatores de risco para a síndrome são agregação de excesso de peso ou adiposidade central, hipertensão arterial, elevação dos triglicerídeos, diminuição do colesterol HDL (High Density Lipoprotein) e intolerância à glicose $^{(5)}$. Sua prevalência, no Brasil, é de $2,6 \%$ nos adolescentes, devendo-se, principalmente, à inatividade física e à alimentação inadequada, assim mostrando a necessidade de intervenções para melhora do quadro e reversão desse problema de saúde pública na adolescência( ${ }^{(6)}$.

Considerando que a instalação precoce dos componentes da SM aumenta os riscos de doenças na vida adulta, a identificação de medidas antropométricas simples, de baixo custo

e não invasivas, que se associem com esses fatores em adolescentes saudáveis é de grande utilidade para a prevenção de doenças crônicas não transmissíveis na vida atual e futura ${ }^{(7)}$. 
Dessa forma, surge a necessidade de explorar as estratégias de promoção da saúde, na tentativa de ampliar o horizonte do processo saúde e doença. A difusão de informações e conhecimentos promove no ser humano a reflexão crítica de sua realidade, favorecendo a vivência de atitudes e comportamentos saudáveis, que garantem a otimização das ações de autocuidado( ${ }^{(8)}$.

Diante do exposto, objetivou-se, no presente estudo, predizer a síndrome metabólica em adolescentes a partir de indicadores antropométricos, metabólicos e hemodinâmicos por análise de sensibilidade e especificidade.

\section{MÉTODOS}

Estudo transversal, realizado no período de julho de 2015 a março de 2016, com 186 adolescentes de oito escolas particulares do município de Picos, Piauí. Picos é uma cidade da região Centro-Sul do Piauí, distante $321 \mathrm{~km}$ da capital, Teresina, que teve uma população estimada de 78.002 pessoas em 2018, com uma extensão territorial de $577,304 \mathrm{~km}^{2(9)}$.

Para esse recorte, obteve-se a amostra a partir de cálculo amostral com a utilização da fórmula para estudos transversais com população finita, que envolve: tamanho da amostra, coeficiente de confiança, tamanho da população, erro amostral absoluto, porcentagem complementar e proporção de ocorrência do fenômeno em estudo(10).

Consideraram-se como parâmetros o coeficiente de confiança de $95 \%(1,96)$, o erro amostral de $3 \%$, a população de 2250 adolescentes (10 a 19 anos) $(\mathrm{N}=2250)$ regularmente matriculados nas escolas da zona urbana e a prevalência de $5 \%$ para SM $(p=0,05)^{(11)}$.

Selecionou-se a amostra de forma aleatória, por meio de um programa de sorteio aleatório (True Random Generator $\left.{ }^{\circledast}\right)$, em que todos os adolescentes tinham a mesma chance de serem selecionados desde que atendessem aos critérios de inclusão (idade entre 10 e 19 anos, regularmente matriculados e que frequentam as aulas) e de exclusão (ter cardiopatia prévia, ser adotado, não estar em jejum de 12 horas).

Selecionaram-se os adolescentes de maneira proporcional à quantidade de alunos matriculados em cada escola participante. Desenvolveu-se o estudo nas escolas particulares por considerar que a literatura tem mostrado ${ }^{(12)}$ que os fatores de risco cardiometabólicos (excesso de peso corporal e sedentarismo) estão ainda mais presentes entre adolescentes de nível socioeconômico mais elevado e, por isso, necessitam que ações de promoção da saúde, de prevenção e de detecção precoce dessas alterações sejam também desenvolvidas para esse público-alvo.

Realizou-se a coleta de dados nas dependências das escolas, em sala reservada para tal, de maneira individual e em um único dia, com exceção da amostra de sangue, pois necessitava de jejum prévio.

Consideraram-se, para este estudo, indicadores antropométricos (índice de massa corporal (IMC), circunferência da cintura (CC), relação cintura-estatura (RCE), índice de conicidade (IC) e circunferência do pescoço (CP), indicadores metabólicos (triglicerídeos (TG), colesterol total (CT), Low Density Lipoproteins (LDL)-colesterol, High Density Lipoproteins (HDL)-colesterol, insulina e homeostatic model assessment (HOMA)) e indicadores hemodinâmicos (pressão arterial sistólica, diastólica e média).

Para a mensuração do peso e da estatura, utilizaram-se, respectivamente, uma balança da marca G-Tec®, com visor digital, e um estadiômetro da marca Seca®. Calculou-se o IMC a partir da fórmula: peso (quilograma-Kg) / estatura ${ }^{2}$ (metro-m). Na realização das medidas de CC e CP, utilizou-se fita métrica inelástica e flexível de 150 centímetros $(\mathrm{cm})$ de comprimento, com precisão de uma casa decimal. Estando o indivíduo em pé, em superfície plana e lisa, em posição ereta, com abdômen relaxado, braços dispostos ao longo do corpo e os pés juntos, aferiuse a CC. Circundou-se com a fita a parte mais estreita do tronco, entre a última costela e a crista ilíaca. Aferiu-se a CP na altura média do pescoço(13). Realizaram-se três medições, considerando-se a média aritmética dos valores ao final. Obteve-se a RCE pelo quociente entre a CC $(\mathrm{cm})$ e a estatura $(\mathrm{cm})^{(14)}$. Calculou-se o índice de conicidade (IC) ${ }^{(15)}$ com a equação que envolve: circunferência de cintura, peso corporal e estatura.

Verificou-se a pressão arterial pelo método auscultatório clássico, com aparelho testado para a pesquisa. A verificação da pressão arterial seguiu os procedimentos recomendados nas VII Diretrizes Brasileiras de Hipertensão $(2016)^{(16)}$. Utilizaram-se manguitos de tamanho apropriado à circunferência dos braços das crianças e dos adolescentes, e desenvolveu-se um protocolo para verificação da pressão arterial que considerou as médias de duas medidas de pressão arterial sistólica (PAS) e pressão arterial diastólica (PAD), aferidas no adolescente após 5 minutos de descanso. No caso de a diferença entre a primeira e a segunda medidas da PAS ou PAD ser maior que 5 milímetros de mercúrio $(\mathrm{mmHg})$, realizou-se uma terceira medida, considerando-se a média entre a segunda e a terceira medidas $^{(17)}$. Calculou-se, ainda, a pressão arterial média (PAM), visto que essa representa PAS e PAD em uma única variável, a partir da aplicação da seguinte fórmula: PAM = [(PAS-PAD/3) + PAD $]^{(18)}$. 
Após um jejum noturno de 12 horas, realizou-se uma coleta de sangue venoso nas dependências das escolas para posterior análise bioquímica. As amostras eram acondicionadas em tubos a vácuo, com gel separador e sem anticoagulante ${ }^{(19)}$. Dosaram-se os TG e a glicose sanguínea utilizando um kit enzimático colorimétrico processado no aparelho Autohumalyzer A5 (Human-2004) ${ }^{(19)}$. Para a dosagem de insulina, utilizou-se o Automated Chemiluminescence System ACS-180 (Ciba-Corning Diagnostic Corp. 1995, EUA) ${ }^{(20)}$.

O diagnóstico da SM na presente amostra definiu-se como sendo a ocorrência de três ou mais dos seguintes critérios: obesidade ( $C \mathrm{C} \geq$ percentil 90 para sexo e idade), dislipidemia (TG > $130 \mathrm{mg} / \mathrm{dl}$; HDL-colesterol < $40 \mathrm{mg} /$ dl), pressão arterial elevada (pressão arterial $\geq$ percentil 90 para sexo, idade e altura) e glicemia de jejum alterada (glicemia $\geq 110 \mathrm{mg} / \mathrm{dl})^{(21)}$.

Categorizaram-se as análises por faixa etária e sexo, dividindo-se a faixa etária em adolescência inicial (10 a 14 anos) e tardia (15 a 19 anos) $)^{(2)}$, e o sexo em masculino e feminino.

Inicialmente, utilizou-se o teste T para amostras independentes para comparação das médias dos indicadores entre os adolescentes com e sem SM, com nível de significância de $p<0,05$. Nesse momento, selecionaram-se todos os indicadores que apresentaram relação significante $(p<0,05)$.

Para a seleção dos pontos de corte, adotou-se a análise a partir da técnica das curvas receiver operating characteristic $(\mathrm{ROC})^{(23)}$, utilizando-se os valores com sensibilidade e especificidade mais próximos entre si, e não inferiores a $60 \%{ }^{(23)}$. Verificou-se a significância estatística de cada análise pela área sob a curva (AUC) ROC e pelo intervalo de confiança a 95\% (IC95\%). Nesse estudo, considerou-se o indicador um bom preditor quando este apresentou área sob a curva ROC maior que 0,50 . Realizou-se a análise estatística dos dados por meio do programa Statistical Package for the Social Sciences (SPSS), versão 20.0.

Os pais/responsáveis pelos avaliados assinaram o Termo de Consentimento Livre e Esclarecido autorizando a participação dos adolescentes. Além disso, os adolescentes assinaram o Termo de Assentimento Livre e Esclarecido. Entregaram-se os resultados dos exames aos adolescentes, com orientação escrita sobre a implementação de hábitos saudáveis de vida, e encaminharam-se os adolescentes que apresentaram alterações nos parâmetros avaliados à unidade básica de saúde da área adstrita de suas residências. O projeto da pesquisa recebeu aprovação pelo Comitê de Ética em Pesquisa da Universidade Federal do Piauí sob o Parecer nº 353.372.

\section{RESULTADOS}

Realizou-se o estudo com adolescentes do semiárido piauiense, obtendo-se a prevalência de indivíduos do sexo feminino $(61,8 \%, n=114)$, com idade entre 15 a 19 anos $(57,5 \%, n=106)$. Identificou-se a SM em $2,7 \%(n=5)$ da amostra conforme disposto na Tabela I.

Tabela I - Caracterização dos adolescentes de 10 a 19 anos de escolas particulares. Picos, Piauí, Brasil, 2015.

\begin{tabular}{lcc}
\hline Variáveis & $\mathbf{n}$ & $\%$ \\
\hline Sexo & 71 & 38,2 \\
$\quad$ Masculino & 115 & 61,8 \\
$\quad$ Feminino & & \\
Idade (em anos) & 79 & 42,5 \\
10-14 & 107 & 57,5 \\
$15-19$ & & \\
Síndrome metabólica & 5 & 2,7 \\
Sim & 181 & 97,3 \\
Não & & \\
\hline
\end{tabular}

n: Número absoluto; \%: Percentual

Os resultados quanto à SM e aos indicadores antropométricos, metabólicos e hemodinâmicos dos adolescentes encontram-se na Tabela II. Constatou-se que a CC, a CT, o HDL-colesterol, a insulina, o índice HOMA, a PAS e a PAM apresentaram estatística significante com a presença da SM. 
Tabela II - Síndrome metabólica e indicadores antropométricos, metabólicos e hemodinâmicos em adolescentes de escolas particulares. Picos, Piauí, Brasil, 2015.

\begin{tabular}{|c|c|c|c|c|}
\hline \multirow{2}{*}{ Indicadores } & \multicolumn{4}{|c|}{ Síndrome metabólica } \\
\hline & Amostra total & Sim & Não & p-valor \\
\hline \multicolumn{5}{|c|}{ Antropométricos, em média (desvio padrão) } \\
\hline $\mathrm{IMC}^{+}$ & $20,94(3,52)$ & $23,95(7,30)$ & $20,86(3,36)$ & 0,399 \\
\hline Circunferência de cintura & $69,66(10,02)$ & $79,88(12,99)$ & $69,38(9,82)$ & $0,021^{\ddagger}$ \\
\hline Relação cintura estatura & $0,43(0,06)$ & $0,47(0,07)$ & $0,43(0,05)$ & 0,099 \\
\hline Índice de conicidade & $11,02(1,14)$ & $11,67(0,16)$ & $11,00(1,15)$ & 0,198 \\
\hline Circunferência de pescoço & $31,40(4,42)$ & $31,00(11,54)$ & $31,41(4,13)$ & 0,939 \\
\hline \multicolumn{5}{|c|}{ Metabólicos, em média (desvio padrão) } \\
\hline Triglicerídeos & $82,37(44,88)$ & $95,38(38,63)$ & $82,01(45,08)$ & 0,513 \\
\hline Glicemia & $72,49(9,93)$ & $76,44(23,99)$ & $72,38(9,39)$ & 0,725 \\
\hline Colesterol total & $154,02(30,16)$ & $123,06(20,84)$ & $154,87(29,96)$ & $0,020^{\ddagger}$ \\
\hline HDL $\$$ & $48,25(8,53)$ & $37,62(3,15)$ & $48,54(8,44)$ & $0,004^{\ddagger}$ \\
\hline LDL" & $89,18(27,28)$ & $66,36(17,39)$ & $89,81(27,27)$ & 0,058 \\
\hline Insulina & $16,04(15,19)$ & $36,56(36,32)$ & $15,47(13,99)$ & $0,002^{\ddagger}$ \\
\hline HOMAT & $2,84(3,01)$ & $8,28(10,65)$ & $2,69(2,44)$ & $0,001^{* *}$ \\
\hline \multicolumn{5}{|c|}{ Hemodinâmicos, em média (desvio padrão) } \\
\hline Pressão arterial sistólica & $108,51(10,65)$ & $124,60(11,48)$ & $108,07(10,30)$ & $0,001^{* *}$ \\
\hline $\mathrm{PAD}^{+1}$ & $69,93(8,77)$ & $76,40(10,04)$ & $69,75(8,69)$ & 0,095 \\
\hline Pressão arterial média & $82,79(8,12)$ & $92,46(5,63)$ & $82,52(8,02)$ & $0,007^{\ddagger \ddagger}$ \\
\hline
\end{tabular}

"p-valor; †IMC: Índice de massa corporal; †Teste T de Student; \$HDL: High Density Lipoprotein (proteína de alta densidade); "LDL: Low Density Lipoprotein (proteína de baixa densidade); ${ }^{\top H O M A: ~ H o m e o s t a ́ t i c a ~ m o d e l ~ A s s e s s m e n t ; ~}{ }^{* *} \mathrm{p} \leq 0,001 ;{ }^{\dagger+P A D: ~ P r e s s a ̃ o ~}$ arterial diastólica; ${ }^{\ddagger 1} p<0,05$

As áreas sob a curva ROC, com os respectivos IC, referentes aos indicadores antropométricos, metabólicos e hemodinâmicos na predição da SM, estão descritas na Tabela III.

Com relação à amostra total, o IMC, CC, RCE, CT, LDL, insulina e índice HOMA não demonstraram poder discriminatório significativo da SM $(\mathrm{Li}-\mathrm{IC}<0,50)$. Por outro lado, quando analisada a área sob a curva ROC, o indicador antropométrico IC demonstrou-se preditor significativo da SM (AUC =0,83). Adicionalmente, o indicador metabólico HDL (AUC $=0,88)$ e os indicadores hemodinâmicos PAS (AUC $=0,86)$ e PAM $(A \cup C=0,84)$ revelaramse com poder discriminatório significativo na predição da $S M(L i-I C \geq 0,50)$.

As adolescentes do sexo feminino de 10 a 14 anos não apresentaram SM, por isso não se realizou análise da curva ROC. Além dos dados apresentados na Tabela III, encontrou-se que, entre as adolescentes do sexo feminino de 15 a 19 anos, os indicadores IMC (AUC = 0,98; IC=0,95-1,00), RCE (AUC = 1,00; IC=1,00-1,00) e PAS (AUC = 0,99; IC=0,97-1,00) revelaram-se com poder discriminatório significativo na predição da SM (AUC e Li-IC $\geq 0,50$ ).

Observou-se também entre os adolescentes do sexo masculino de 10 a 14 anos que somente a PAM (AUC = 0,81 ; IC = 0,74-0,95) mostrou-se como bom preditor da SM. Já entre os alunos de 15 a 19 anos, os indicadores PAD ( $A \cup C=0,96 ; I C=0,90-1,00)$ e PAM ( $A \cup C=0,96 ; I C=0,90-1,00)$ revelaram-se com poder discriminatório significativo na predição da SM (AUC e Li-IC $\geq 0,50$ ).

Com relação à amostra total, obtiveram-se os pontos com maior sensibilidade e especificidade: $11,56 \mathrm{~cm}$ para IC, $39,05 \mathrm{mg} / \mathrm{dl}$ para HDL, $121,5 \mathrm{mmHg}$ para PAS e $89,0 \mathrm{mmHg}$ para PAM. Já nas adolescentes do sexo feminino de 15 a 19 anos, consideraram-se os pontos de corte: $27,10 \mathrm{~kg} / \mathrm{m}^{2}$ para IMC, $0,53 \mathrm{~cm}$ para RCE e $124 \mathrm{mmHg}$ para PAS (Tabela IV).

Conforme apresenta a Tabela $\mathrm{V}$, com relação aos adolescentes do sexo masculino de 10 a 14 anos, obteve-se ponto de corte de $89,0 \mathrm{mmHg}$ para PAM; e de $94,66 \mathrm{mmHg}$ nos adolescentes de 15 a 19 anos. 
Tabela III - Área sob a curva ROC* e IC 95\% ${ }^{\dagger}$ entre indicadores antropométricos, metabólicos e hemodinâmicos e SM na amostra. Picos, Piauí, Brasil, 2015.

\begin{tabular}{lc}
\hline Síndrome metabólica & Área sob a curva ROC ${ }^{*}(\mathbf{I C 9 5} \%)^{\dagger}$ amostra total \\
\hline Antropométricos & - \\
IMC & \\
Circunferência de cintura & $0,73(0,43-1,00)$ \\
Relação cintura estatura & - \\
Índice de conicidade & $0,83(0,76-0,90)^{\S}$ \\
Metabólicos & \\
Colesterol total & $0,18(0,15-0,35)$ \\
LDL" & $0,22(0,06-0,39)$ \\
HDL & $0,88(0,82-0,94)^{\S}$ \\
Insulina & $0,69(0,44-0,94)$ \\
HOMA** & $0,66(0,40-0,92)$ \\
Hemodinâmicos & $0,86(0,70-1,00)^{\S}$ \\
Pressão arterial sistólica & - \\
Pressão arterial diastólica & $0,84(0,74-0,95)$ \\
Pressão arterial média & \\
\hline
\end{tabular}

*ROC: Receiver Operating Characteristic; † IC95\%: Intervalo de confiança a 95\%; ‘IMC: Índice de massa corporal; §: Área sob a curva ROC apresentando poder discriminatório da síndrome metabólica (Li-IC $\geq 0,50)$; "LDL: Low Density Lipoprotein (proteína de baixa densidade); "HDL: High Density Lipoprotein (proteína de alta densidade); **HOMA: Homeostatic model assessment; $\%:$ Percentual

Tabela IV - Pontos de corte, sensibilidade e especificidade dos indicadores antropométricos, metabólicos e hemodinâmicos com predição da síndrome metabólica (SM) na amostra. Picos, Piauí, Brasil, 2015.

\begin{tabular}{|c|c|c|c|c|c|c|}
\hline SM & $\begin{array}{c}\text { PC- amostra } \\
\text { total }\end{array}$ & $\begin{array}{c}S(\%) \\
\text { amostra total }\end{array}$ & $\begin{array}{c}E(\%) \text { amostra } \\
\text { total }\end{array}$ & $\begin{array}{c}\text { PC- } 15 \text { a } 19 \\
\text { anos }\end{array}$ & $\begin{array}{c}S(\%) 15 \text { a } \\
19 \text { anos }\end{array}$ & $\begin{array}{c}E(\%) 15 \text { a } 19 \\
\text { anos }\end{array}$ \\
\hline \multicolumn{7}{|l|}{ Antropométricos } \\
\hline IMC* & $\mathrm{NP}^{\dagger}$ & - & - & 27,10 & 100,0 & 98,5 \\
\hline RCE & $N P^{\dagger}$ & - & - & 0,53 & 100,0 & 100,0 \\
\hline Índice de conicidade & 11,56 & 80,0 & 81,2 & $\mathrm{NP}^{\dagger}$ & - & - \\
\hline \multicolumn{7}{|l|}{ Metabólicos } \\
\hline $\mathrm{HDL}^{\ddagger}$ & 39,05 & 100,0 & 82,9 & $\mathrm{NP}^{\dagger}$ & - & - \\
\hline \multicolumn{7}{|l|}{ Hemodinâmicos } \\
\hline PAS & 121,5 & 80,0 & 88,4 & 124,0 & 100,0 & 98,5 \\
\hline PAM & 89,0 & 80,0 & 79,6 & $\mathrm{NP}^{\dagger}$ & - & - \\
\hline
\end{tabular}

SM: Síndrome metabólica; PC: Ponto de corte; S: Sensibilidade; E: Especificidade; *IMC: Índice de massa corporal; RCE: Relação cintura estatura; ${ }^{\dagger} \mathrm{NP}$ : Não preditor; ${ }^{\ddagger} \mathrm{HDL}$ : High Density Lipoprotein (proteína de alta densidade); PAS: Pressão arterial sistólica; PAM: Pressão arterial média; \%: percentual

Tabela V - Pontos de corte, sensibilidade e especificidade dos indicadores antropométricos, metabólicos e hemodinâmicos com predição da síndrome metabólica (SM) em alunos de escolas particulares. Picos, Piauí, Brasil, 2015.

\begin{tabular}{|c|c|c|c|c|c|c|}
\hline SM & $\begin{array}{c}\mathrm{PC}-10 \text { a } 14 \\
\text { anos }\end{array}$ & $\begin{array}{c}S(\%) 10 \text { a } 14 \\
\text { anos }\end{array}$ & $\begin{array}{c}E(\%) 10 \text { a } \\
14 \text { anos }\end{array}$ & $\begin{array}{c}\text { PC-15 a } 19 \\
\text { anos }\end{array}$ & $\begin{array}{c}S(\%) 15 \text { a } 19 \\
\text { anos }\end{array}$ & $\begin{array}{c}E(\%) 15 \text { a } 19 \\
\text { anos }\end{array}$ \\
\hline Antropométricos & $\mathrm{NP}^{*}$ & - & - & $N P^{*}$ & - & - \\
\hline Metabólicos & $N P^{*}$ & - & - & $N P^{*}$ & - & - \\
\hline \multicolumn{7}{|l|}{ Hemodinâmicos } \\
\hline PAD & $N P^{*}$ & - & - & 81,5 & 100,0 & 92,1 \\
\hline PAM & 89,0 & 100,0 & 79,3 & 94,66 & 100,0 & 92,1 \\
\hline
\end{tabular}

SM: Síndrome metabólica; PC: Ponto de corte; S: Sensibilidade; E: Especificidade; PAD: Pressão arterial diastólica; PAM: Pressão arterial média; *NP: Não preditor; \%: Percentual 


\section{DISCUSSÃO}

Obteve-se a prevalência de síndrome metabólica na população estudada de 2,7\% $(n=5)$, resultado semelhante ao encontrado no Estudo de Riscos Cardiovasculares em Adolescentes (ERICA), primeiro de âmbito nacional e de base escolar no Brasil, que encontrou uma prevalência de 2,6\%, pouco superior à encontrada em Teresina, Piauí $(1,9 \%)^{(24)}$. Entretanto, em outros estudos, a prevalência variou entre $4,1 \%^{(9,22)}$ e $14,1 \%{ }^{(24)}$. Em um estudo desenvolvido na Guatemala, com 302 crianças/adolescentes, constatou-se a prevalência de SM de 2,0\%, entretanto, em aproximadamente $54 \%$ dos participantes, verificou-se pelo menos um componente da $S^{(25)}$. Já em um estudo desenvolvido na Coreia, com 1554 adolescentes de 10 a 19 anos, constatou-se a prevalência de SM de 2,3\% na amostra total, de $2,8 \%$ em adolescentes com sobrepeso e de $23,7 \%$ em adolescentes obesos ${ }^{(26)}$.

A heterogeneidade de definições e de pontos de corte para os componentes da SM podem explicar, ao menos em parte, as diferentes prevalências relatadas na literatura. Ainda assim, admite-se que estas vêm aumentando, tanto em países desenvolvidos quanto naqueles em desenvolvimento ${ }^{(27)}$. Além disso, quando a SM é investigada entre adolescentes com sobrepeso e/ou obesidade, a prevalência aumenta de maneira significativa $(p<0,05)$, com prevalências que variaram entre $11,3 \%$ a $31,8 \%$, de acordo com os municípios brasileiros, sendo que $21,3 \%$ dos adolescentes obesos brasileiros apresentaram $\mathrm{SM}^{(24)}$. Os achados deste estudo corroboraram essa informação quando se observou que o IMC apresenta-se como um bom preditor da SM entre adolescentes do sexo feminino de 15 a 19 anos.

A análise da sensibilidade e da especificidade por meio da construção de curvas ROC não só permite a identificação do melhor ponto de corte, como também fornece a área sob a curva, que traduz o poder de discriminação de um indicador para um determinado desfecho ${ }^{(28)}$.

Estudos têm demonstrado que a CC em adolescentes constitui um bom indicador do conteúdo de gordura abdominal visceral e de fatores de risco cardiovasculares, podendo ser um instrumento simples para identificar SM ${ }^{(13)}$.

Evidenciou-se, no presente estudo, boa sensibilidade e especificidade para a predição da SM, tanto o IC para a amostra total, quanto a RCE para alunas de 15 a 19 anos, com pontos de corte de $11,56 \mathrm{~cm}$ e $0,53 \mathrm{~cm}$ respectivamente. Observou-se também na presente pesquisa que os adolescentes com SM apresentaram menor média de HDL e maiores médias de CT, de insulina e índice HOMA, e apenas o HDL mostrou-se como bom preditor para triagem da SM na amostra total.

O aumento do HDL permite redução do colesterol na circulação sanguínea e nos tecidos periféricos. Além disso, possui ação antioxidante, anti-inflamatória, anticoagulante e de proteção endotelial. Em contrapartida, baixas concentrações de HDL também aumentam o risco para o desenvolvimento de doenças cardiovasculares ${ }^{(29)}$.

A baixa concentração de HDL no presente estudo pode ser explicada pelo processo de transição nutricional vivenciado no Brasil, em que o consumo alimentar tem sido principalmente constituído de alimentos ultraprocessados e de alto teor energético. Entre os adolescentes, destaca-se a frequência elevada de consumo de biscoitos recheados, linguiça, salsicha, mortadela, sanduíches e salgados, e o baixo consumo alimentar de frutas e verduras ${ }^{(30)}$.

Com relação aos indicadores hemodinâmicos, no estudo em questão, a PAS e a PAM mostraram-se bons preditores da SM na amostra total. Quando categorizados por sexo e idade, a PAS mostrou-se preditora em alunas de 15 a 19 anos; a PAM mostrou-se preditora em adolescentes do sexo masculino, de ambas as faixas etárias, e PAD mostrou-se preditora para alunos de 15 a 19 anos.

Encontraram-se resultados semelhantes em um estudo desenvolvido com crianças e adolescentes em Guabiruba, Santa Catarina ${ }^{(31)}$, onde os estudantes com SM apresentaram elevação dos valores da PA sistólica e da PA diastólica em comparação àqueles sem SM $(p<0,0001)$. No estudo $\mathrm{ERICA}^{(24)}$, encontrou-se a prevalência de elevação da PA de $8,2 \%$ entre os adolescentes estudados. Além disso, 57,6\% dos adolescentes com SM tinham PA elevada, 0 que ratifica a PA como um componente importante de ser avaliado no período da adolescência.

Considerando-se que a hipertensão arterial sistêmica (HAS) do adulto pode ter a sua origem na infância e na adolescência, estratégias preventivas, notadamente relacionadas à identificação dos diversos fatores de risco cardiovascular associados com a HAS nessa faixa etária, devem ser adotadas precocemente, na tentativa de reduzir as complicações tardias dessa moléstia ${ }^{(29)}$.

Diante do exposto, ressalta-se que as ações de promoção à saúde por parte dos profissionais de saúde, em especial os da atenção básica, devem ter forte caráter promotor de saúde, com estímulo à criação de hábitos e ambientes saudáveis, e essas ações devem ser desenvolvidas para assistir crianças e adolescentes com vistas a Ihes proporcionar condições que favoreçam o seu desenvolvimento saudável e pleno ${ }^{(32)}$. 
Aponta-se como uma limitação do presente estudo a verificação da pressão arterial. Embora tenha sido verificada em duplicata e seguindo todas as recomendações das diretrizes de hipertensão arterial, verificou-se a PA em um único momento. Sugere-se, assim, que outros estudos sejam realizados com uma avaliação de PA em três dias alternados, conforme recomendações.

\section{CONCLUSÃO}

Os indicadores antropométricos, metabólicos e hemodinâmicos demonstraram-se preditores da síndrome metabólica, merecendo destaque o índice de conicidade, a lipoproteína de alta densidade, a pressão arterial sistólica e a pressão arterial média.

FONTE FINANCIADORA: Fundação de Amparo a Pesquisa do Estado do Piauí - FAPEPI.

\section{CONTRIBUIÇÕES}

Thais Norberta Bezerra de Moura contribuiu na concepção e projeto, na análise e interpretação dos dados, na redação do artigo e na revisão crítica relevante do conteúdo intelectual. Jéssica Denise Vieira Leal, Gisely Silva Sousa e Regianne Kellyne Carneiro de Sousa contribuíram na análise e interpretação dos dados e na redação do artigo. Edina Araújo Rodrigues Oliveira contribuiu na análise e interpretação dos dados, na redação do artigo e na revisão crítica do conteúdo intelectual. Luisa Helena de Oliveira Lima contribuiu na concepção e projeto, na análise e interpretação dos dados, na redação do artigo, na revisão crítica do conteúdo intelectual e na aprovação da versão final a ser publicada.

\section{REFERÊNCIAS}

1. Ribeiro AC, Padoin SMM, Paula CC, Terra MG. The daily living of adolescentes with HIV/Aids: impersonality and tendency to fear. Text Context Nursing [Internet]. 2013 [acesso 2018 Set 15];22(3):680-6. Disponível em: http://www.scielo.br/pdf/tce/v22n3/en_v22n3a14.pdf

2. Pereira AMVB, Gomes I, Schwanke CHA. Metabolic syndrome in elderly assisted in tertiary health care in Curitiba, Paraná, Brazil: prevalence and association with health, functional capacity, life style, and sociodemographic factors. Sci Med [Internet]. 2016 [acesso em 2018 Set 08];26(3):1-9. Disponível em: http://revistaseletronicas. pucrs.br/ojs/index.php/scientiamedica/article/view/23444/14874

3. Leitão MP, Martins IS. Prevalence and factors associated with metabolic syndrome in users of primary healthcare units in São Paulo - SP. Rev Assoc Med Bras [Internet]. 2012 [acesso em 2018 Ago 15];58(1):60-9. Disponível em: http://www.scielo.br/pdf/ramb/v58n1/v58n1a16.pdf.

4. Araújo MFM, Freitas RWJF, Lima ACS, Pereira DCR, Zanetti ML, Damasceno MMC. Relation between sleep quality and metabolic syndrome among university students. Texto \& Contexto Enferm [Internet]. 2015 [acesso em 2018 Set 08];24(2):505-12. Disponível em: www.scielo.br/pdf/tce/v24n2/pt_0104-0707-tce-24-02-00505.pdf

5. Silva ARV. Risk factors for metabolic syndrome in adolescents [editorial]. Rev Enferm UFPI [Internet]. 2014 [acesso em 2018 Ago 15];3(2):1-3. Disponível em: http://www.ojs.ufpi.br/index.php/reufpi

6. Barreto AC Neto, Andrade MIS, Lima VLM, Diniz AS. Peso corporal e escores de consumo alimentar em adolescentes no nordeste brasileiro. Rev Paul Pediatr [Internet]. 2015 [acesso em 2018 Set 08];33(3):318-25. Disponível em: http://www.scielo.br/pdf/rpp/v33n3/0103-0582-rpp-33-03-0318.pdf

7. Faria FR, Faria ER, Faria FR, Paula HAA, Franceschini SCC, Priore SE. Association between metabolic syndrome and anthropometric and body composition indicators in adolescents. Rev Assoc Bras Nutrição [Internet]. 2014 [acesso em 2018 Set 08];6(1):13-20. Disponível em: https://www.rasbran.com.br/rasbran/article/view/163/123

8. Faial LCM, Silva RMCRA, Pereira ER, Refrande SM, Souza LMC, Faial CSG. The school as an environment for health promotion during adolescence: literature review. Rev Pró-UniverSUS [Internet]. 2016 [acesso em 2018 Set 15];7(2):22-9. Disponível em: http://editora.universidadedevassouras.edu.br/index.php/RPU/article/ view/344/525

9. Instituto Brasileiro de Geografia e Estatística, Diretoria de Pesquisas, Coordenação de População e Indicadores Sociais. Estimativas da população residente com data de referência 2017. [Internet]. 2017 [acesso em 
2018 Set 08]. Disponível em: https://www.ibge.gov.br/estatisticas-novoportal/por-cidade-estado-estatisticas. $\mathrm{html} ? \mathrm{t}=$ destaques\&c=2208007

10. Miot HA. Sample size in clinical and experimental trials. J Vasc Bras [Internet]. 2011 [acesso em 2018 Ago 15];10(4):275-8. Disponível em: http://www.scielo.br/pdf/jvb/v10n4/en_v10n4a01

11. Marcarini M, Mendes GK. Metabolic syndrome and its relationship with the nutritional status inadolescents variability of diagnostic criteria. Scientia Medica [Internet]. 2013 [acesso em 2018 Ago 16];23(2):108-18. Disponível em: http://revistaseletronicas.pucrs.br/ojs/index.php/scientiamedica/article/view/12819/9661

12. Ferreira RW, Rombaldi AJ, Ricardo LIC, Hallal PC, Azevedo MR. Prevalence of sedentary behavior and its correlates among primary and secondary school students. Rev Paul Pediatr [Internet]. 2016 [acesso em 2018 Ago 16];34(1):56-63. Disponível em: http://www.scielo.br/pdf/rpp/v34n1/0103-0582-rpp-34-01-0056.pdf

13. Ferreira MG, Valente JG, Gonçalves-Silva RMV, Sichieri R. Accuracy of waist circumference and waist-to-hip ratio as predictors of dyslipidemia in a cross-sectional study among blood donors in Cuiabá, Mato Grosso State, Brazil. Cad Saúde Pública [Internet]. 2006 [acesso em 2018 Ago 16];22(2):307-14. Disponível em: http://www. scielo.br/pdf/csp/v22n2/08.pdf

14. Pereira PF, Serrano HMS, Carvalho GQ, Lamounier JA, Peluzio MCC, Franceschini SCC, et al. Waist and waistto-height ratio: useful to identify the metabolic risk of female adolescents? Rev Paul Pediatr [Internet]. 2011 [acesso em 2018 Ago 16];29(3):372-7. Disponível em: http://www.scielo.br/pdf/rpp/v29n3/en_a11v29n3.pdf

15. Pitanga FJG, Lessa I. Sensitivity and specificity of the conicity index as a coronary risk predictor among adults in Salvador, Brazil. Rev Bras Epidemiol [Internet]. 2004 [acesso em 2018 Ago 16];7(3):259-69. Disponível em: http://www.scielo.br/pdf/rbepid/v7n3/04.pdf

16. Malachias MVB, Souza WKSB, Plavnik FL, Rodrigues CIS, Brandão AA, Neves MFT, et al. $7^{\text {th }}$ Brazilian Guideline of Arterial Hypertension. Arq Bras Cardiol [Internet]. 2016 [acesso em 2018 Ago 16];107(3 Supl 3):1-83. Disponível em: http://www.scielo.br/pdf/abc/v107n3s3/0066-782X-abc-107-03-s3-0000.pdf

17. Molina MCB, Faria CP, Montero MP, Cade NV, Mill JG. Cardiovascular risk factors in 7-to-10-year-old children in Vitória, Espírito Santo State, Brazil. Cad Saúde Pública [Internet]. 2010 [acesso em 2018 Ago 16];26(5):909-17. Disponível em: http://www.scielo.br/pdf/csp/v26n5/13.pdf

18. Silva JLN, Silva FL Júnior, Ferreira AP, Simões HG. Characterization and influence of indicators of central obesity, fitness cardiorespiratory and level of physical activity on blood pressure of school. Rev Andal Med Deporte [Internet]. 2017 [acesso em 2018 Ago 16];10(1):25-30. Disponível em: www.elsevier.es/pt-revistarevista-andaluza-medicina-del-deporte-284-pdf-S1888754616300089-S300

19. Brito BB, Leal JDV, Formiga LMF, Frota KMG, Silva ARV, Lima LHO. Cardiovascular diseases: risk factors in adolescents. Cogitare Enferm [Internet]. 2016 [acesso em 2018 Ago 16];21(2):1-8. Disponível em: http://www. saude.ufpr.br/portal/revistacogitare/wp-content/uploads/sites/28/2016/10/41848-177492-1-PB.pdf

20. Gauche R, Gadelha AB, Paiva FML, Oliveira PFA, Lima RM. Strength, muscle quality and markers of cardiometabolic risk in older women. Rev Bras Cineantropom Desempenho Hum [Internet]. 2015 [acesso em 2018 Ago 16];17(2):18694. Disponível em: https://periodicos.ufsc.br/index.php/rbcdh/article/download/1980-0037.2015v17n2p186/28728

21. Cook S, Weitzman M, Auinger P, Nguyen M, Dietz WH. Prevalence of a metabolic syndrome phenotype in adolescents: findings from the third National Health and Nutrition Examination Survey, 1988-1994. Arch Pediatr Adolesc Med [Internet]. 2003 [acesso em 2018 Ago 16];157(8):821-7. Disponível em: https://jamanetwork.com/ journals/jamapediatrics/fullarticle/481403

22. Unicef. Fase inicial e fase final da adolescência [Internet]. 2011 [acesso em 2018 Set 15]. Disponível em: http:// www.unicef.org/brazil/sowc2011/foco1.html

23. Erdreich LS, Lee ET. Use of relative operating characteristic analysis in epidemiology: method for dealing with subjective judgement. Am J Epidemiol [Internet]. 1981 [acesso em 2018 Ago 16];114(5):649-62. Disponível em: https://doi.org/10.1093/oxfordjournals.aje.a113236

24. Kuschnir MCC, Bloch KV, Szklo M, Klein CH, Barufaldi LA, Abreu GA, et al. ERICA: prevalence of metabolic syndrome in Brazilian adolescents. Rev Saúde Pública [Internet]. 2016 [acesso em 2018 Ago 16];50(Supl 1):11s. Disponível em: http://www.scielo.br/pdf/rsp/v50s1/0034-8910-rsp-S01518-87872016050006701.pdf 
25. Pontes LM, Amorim RJM, Lira PIC. Components of metabolic syndrome and associated factors in adolescents: a case-control study. Rev AMRIGS [Internet]. 2016 [acesso em 2018 Ago 16];60(2):121-8. Disponível em: http:// www.amrigs.org.br/revista/60-02/10_1598_Revista\%20AMRIGS.PDF

26. Mbowe O, Diaz A, Wallace J, Mazariegos M, Jolly P. Prevalence of metabolic syndrome and associated cardiovascular risk factors in Guatemalan school children. Matern Child Health J [Internet]. 2014 [acesso em 2018 Ago 16];18(7):1619-27. Disponível em: http://europepmc.org/articles/PMC4055521

27. Park SH, Park JH, Kang JW, Park HY, Park J, Shin KJ. Prevalence of the metabolic syndrome and abnormal lipid levels among Korean adolescents. J Paediatr Child Health [Internet]. 2013 [acesso em 2018 Ago 16];49(7):5827. Disponivel em: https://doi.org/10.1111/jpc.12284

28. Ribeiro-Silva RC, Florence TCM, Conceição-Machado MEP, Fernandes GB, Couto RD. Anthropometric indicators for prediction of metabolic syndrome in children and adolescents: a population-based study. Rev Bras Saude Mater Infant [Internet]. 2014 [acesso em 2018 Ago 16];14(2):173-81. Disponível em: http://www.scielo.br/pdf/ rbsmi/v14n2/1519-3829-rbsmi-14-02-0173.pdf

29. Özer S, Yılmaz R, Özlem KN, Sönmezgöz E, Karaaslan E, Altuntaş B, et al. Higher hdl levels are a preventive factor for metabolic syndrome in obese Turkish children. Nutr Hosp [Internet]. 2014 [acesso em 2018 Ago 16];31(1):307-12. Disponível em: https://www.researchgate.net/publication/270655893/download

30. Pereira PF, Faria FR, Faria ER, Hermsdorff HHM, Peluzio MCG, Franceschini SCC, et al. Anthropometric indices to identify metabolic syndrome and hypertriglyceridemic waist phenotype: a comparison between the three stages of adolescence. Rev Paul Pediatr [Internet]. 2015 [acesso em 2018 Ago 16];33(2):194-203. Disponível em: http://www.scielo.br/pdf/rpp/v33n2/0103-0582-rpp-33-02-00194.pdf

31. Rosini N, Moura SAZO, Rosini RD, Machado MJ, Silva EL. Metabolic syndrome and importance of associated variables in children and adolescents in Guabiruba - SC, Brazil. Arq Bras Cardiol [Internet]. 2015 [acesso em 2018 Ago 16];105(1):37-44. Disponível em: http://www.scielo.br/pdf/abc/v105n1/0066-782X-abc-20150040.pdf

32. Lima LHO. Promoção da saúde na infância e adolescência: perspectivas e desafios. In: Silva ARV, Oliveira AKS, Lima LHO, Machado ALG. Interlocuções entre vivências coletivas na promoção da saúde [Internet]. Teresina: EDUFPI; 2018 [acesso em 2018 Set 08]. p. 27-30. Disponível em: http://saudecoletivapicos.com.br/media/livros/ Interloc.pdf

\section{Endereço para correspondência:}

Thais Norberta Bezerra de Moura

Força Estadual de Saúde do Maranhão - FESMA

Av. Borborema, Quadra-16, 25

Bairro: Calhau

CEP: 65071-360 - São Luís - MA - Brasil

E-mail: thaisinha_moura@hotmail.com 